\title{
Analysis and Design on Decision Support System of Security Risk Management in Rural Power Network
}

\author{
Dongsheng Zhou ${ }^{1}$ and Tao Yang, ${ }^{2, *}$ \\ ${ }^{1}$ College of Information and Electrical Engineering, \\ Shenyang Agriculture University, Shenyang 110866, China \\ ${ }^{2}$ College of Information and Electrical Engineering, \\ Shenyang Agriculture University, Shenyang 110866, China \\ zhoudsh8021@sina.com, yangtaosx@yahoo.com.cn
}

\begin{abstract}
The paper, according the investigation and research of the requirement of rural grid security risk management, designs a decision support system to assist decision-making of security risk management. System database and knowledge base were built, progress the model of security risk assessment. The application of methods of statistical analysis and reasoning, avoids, prevents and controls the security risk of rural power network effectively. Through system applying in Changchun rural power company, improved the quality and level of decision on security risk management.
\end{abstract}

Keywords: Decision support system, Security risk management, Rural power network.

\section{Introduction}

Security of risk evaluation is an important method of modern management. Promote the security of risk evaluation is a critical step to measure and to predict the system of security risk and control the incident. The implementation of security of risk evaluation can pre-control system of risk, as well as make security management more scientific and increase the technology content of safety management.

With the development of information technology, the construction of information technology in rural grid system has been tremendous development, but information of security of risk management is still in a relatively weak aspect. By designing and applying the decision-supported system of security of risk evaluation, one can avoids, prevents and controls the security of risk in rural grid effectively, and also improve the quality and level of decision of security of risk management.

\section{Summary of Decision Support System}

The conception of decision support system is proposed firstly in 1971 by M. S. Morton, whom comes from the United States. Decision-supported system is a integrated system, which aims decision-making for dealing with problems; it uses decision-making

* Corresponding author.

D. Li, Y. Liu, and Y. Chen (Eds.): CCTA 2010, Part III, IFIP AICT 346, pp. $298-303,2011$.

(C) IFIP International Federation for Information Processing 2011 
resources (data, models, knowledge, etc) to combine and to integrate, builds solutions as well; it is also gradually access the decisions through model calculations, knowledge inference, multidimensional data analysis and program modification.

Decision support system includes traditional decision-supported system, intelligent decision support system, new decision support system and integrated decision support system. Traditional decision-supported system is an aided decision-making system which combined model base and database, and it is the first of decision support system; intelligent decision support system is an aided decision-making system which uses expert system and data mining and based on the traditional decision-supported system; new decision-supported system is a decision support system which uses warehouse of data, online analytical and data mining; integrated decision support system is a more advanced decision support system, it is also the direction of future research in the area of decision-supported system. Decision support system has a wide range of applications in production, management and other aspects, such as agriculture, resource management, business management, manufacturing in production and management, decision-making management of e-government, etc.

\section{System Design}

\subsection{Design Goals}

The system uses the methods of work environment, protection of equipments and machines, quality of personnel, workplace management, integrated production of safety, to assist safe managers to evaluate the security of risks and improve the quality and levels of decision-making.

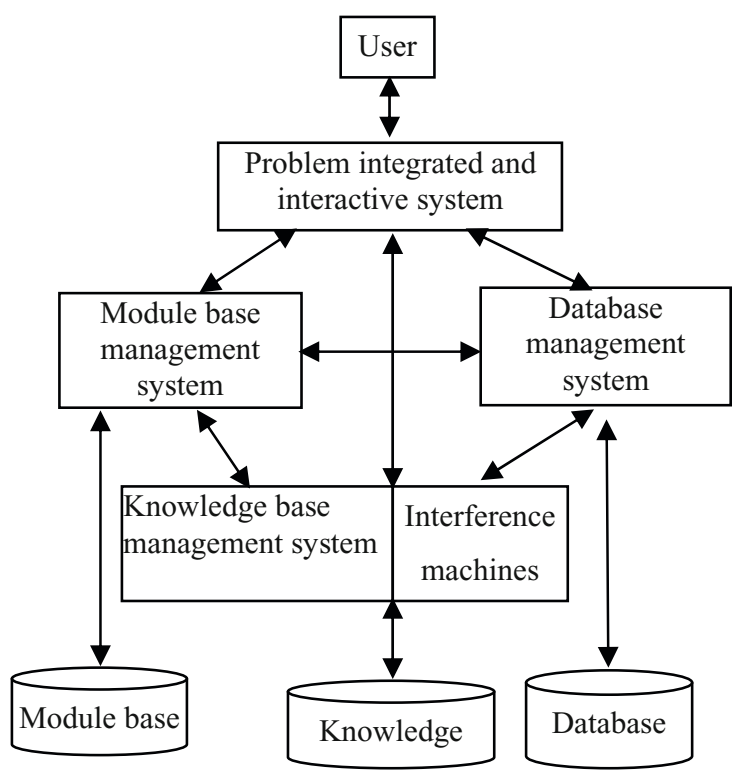

Fig. 1. System Structure 


\subsection{Structure and Function}

\subsubsection{Structure of System}

The structure of the system is consisted of the data of database, knowledge base and module base, the data is reasoned by inference machines and analyzed in the management system of knowledge base, then the result is outputted to human-machine interface, it plays an aided role in the step of decision making for users.

\subsubsection{System Function}

The system is designed for four modules, namely database management system, knowledge base management, model base management decision-aided support.

(1) Database management system. The module managed the basic information of work environment, protection of equipments and machines, quality of personnel, workplace management and integrated production safety.

(2) Knowledge base management system. The module included transport evaluated criteria, transform evaluation criteria and distribution evaluation criteria.

(3) Model base management system. The module includes hazard identification model and safe evaluation model.

(4) Decision-aided support system. This module can aid policy-makers to make decisions by periodic safe and risk assessment and real-time safe and risk assessment.

\subsection{Database Design}

The system chooses Microsoft SQL Server 2005 as the management system of database for backstage, including the design of database, knowledge base and module base.

Database is an important resource of the decision-supported system, a basic part of module base, knowledge base and interface of human-machine. The safe and risk evaluation of management of the power companies are mainly dealt with the evaluation in five aspects: work environment, protection of equipments and machines, quality of personnel, workplace management, integrated production of safety, the module of database management will manage the data together.

Knowledge base is a combination of knowledge, which is stored according to some regulations in the computer, the reliability of decision support system is depend on the quality of knowledge which is stored in the knowledge base. The knowledge base of this research is on the basis of grid standardized regulations of safe management, accompanying with experience of working in many years and against with the actual situation of grid in rural areas, which can also be divided into three aspects: power transport, power transform and power distribution. Each kind of work is classified and given the ID number of everyone, which as the only mark for the task. Every work is listed by the procedure of safe production: before work, work and after work, these procedures evaluate varieties of potential danger as corresponding of ratio and evaluate every procedure for its corresponding mark as well, also, the data will be the critical depends of safe production evaluation. Table 1 is the structure for standard of marks. 


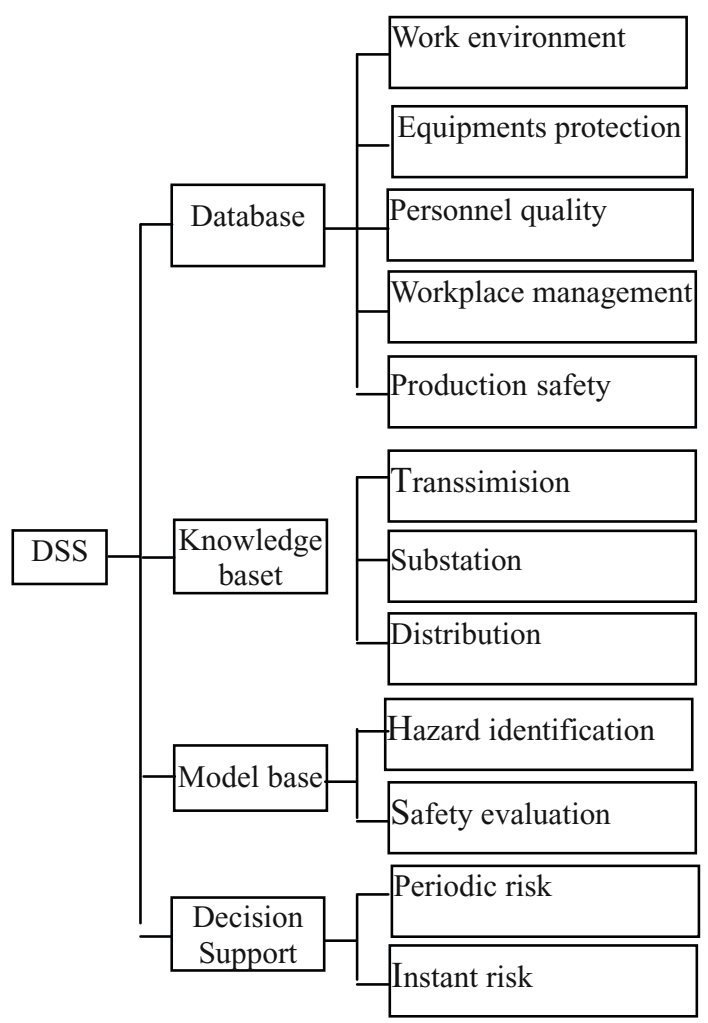

Fig. 2. System functions

Table 1. Table of evaluating marks

\begin{tabular}{ll}
\hline Name of Field & Description of Field \\
\hline StdNumber & Number of Evaluation \\
StdItem & Project of Evaluation \\
StdMethod & Standard and Method of Evaluation \\
StdGrade & Mark of Standard \\
Weight & Ratio \\
Method & Measure of Evaluation \\
Scope & Range of Using \\
Cycle & Period of Evaluation \\
StdSource & Resource of Project Standard \\
\hline
\end{tabular}

Module base is the critical part of decision-supported system, involves database of module and management system of module database. There are two main modules in this system: identified module of dangerous resource and module of integrated evaluation. Identified module of dangerous has important position in the system of management evaluation in safe risk area, which directly affects the exact extent of safe 
evaluation. The system through the method of LEC to identify the dangerous resource, LEC is also called the line-evaluated method of working condition, its module is $D=L \times E \times C$. The size of risking value depend on $L$, which represents feasibility of accident-happened, as well as $E$ (it is the frequency of exposure in the dangerous environment) and $C$ (it is related to the results affect by the existed accident). The module depends on the value of LEC as the evaluation of working in risk conditions, and then takes corresponding steps. Module of integrated evaluation use the method of lined, ratio and average, its module can write as

$$
T=\sum_{i=1}^{p}\left\{\sum_{k=1}^{M}\left[\sum_{j=1}^{k} F_{j} \bullet Q_{j} / N\right] W_{k}\right\} \bullet X_{i}
$$

$P$ represents the category of project with the system of evaluated value; $M$ is the number of evaluated key element, which is related to the standard of evaluation in the order of i; $N$ is the number of person whom attend the evaluation; $W_{k}$ is the ratio of standard of evaluation in the order of $\mathrm{k}$ in the category of $i$; $F_{j}$ is the evaluated mark which evaluated by the standard of the order of $k$; $Q_{j}$ represents the ratio of evaluation standard in the order of $k$ by the person's order of $j ; X_{i}$ is the ratio of standard of evaluation in the order of $i$. By using this method, people can give marks of all kinds of parameters in the safe risk management.

\section{Inference Engine}

Inference Engine gives its judge according to the marks of the former system.

The instant evaluation can not only check all the projects as a whole, but can check single project, the formula is

$$
\rho=\frac{\sum_{k=1}^{n} s_{k} * w_{k}}{S}
$$

$\rho$ is the level of safety; $S_{k}$ is the single mark of safety; $W_{k}$ represents single ratio; $S$ represents the sum-number. In this way, we can calculate the safe level of working:

Safe and controllable $(\rho \geq 0.9)$

Low risks $(0.9>\rho \geq 0.75)$

Risks $(0.75>\rho \geq 0.6)$

High risks $\quad(0.6>\rho \geq 0.4)$

Safety is out of control $(\rho<0.4)$

Period evaluation is a method of statistics and analysis in each work unit. The method can integrated count and analyze the work environment, protection of equipments and machines, quality of personnel, workplace management, integrated production of safety, and then give a result in the period of safe risk evaluation. Managers of production can make decisions by using the data and instruct production. The mark for formula of single task is 


$$
{ }_{s}=\frac{\sum_{k=1}^{n} s_{k} * w_{k}}{n}
$$

$S$ is single work's average mark; $s_{k}$ is mark of single safety; $w_{k}$ represents single ratio; $n$ represents number of working. The sun-mark of evaluated result can calculate by the task mark of single work, its formula is

$$
\bar{S}=\sum_{k=1}^{n} \bar{s}_{k} * w_{k}
$$

$\bar{S}$ represents the sum-mark of evaluated result; $s_{k}$ is the task mark of single work; $W_{k}$ is single ratio. In this way, we can count the sum-mark in the period and obtain the result of single work.

As immediate evaluation has a dynamic wave of data, evaluate once cannot reflect the actual condition objectively, so we need to results of evaluation in many workplace. According to the result of evaluation, decision-makers can analyze the immediate work and safe risk of working in the period. finally, they can make newly decisions.

\section{Conclusion}

This essay analyze the security of grid and the risk management of decision-supported system in rural areas, by combining periodic and real-time evaluation method, simultaneously, by using the method of linear model ratio, which can provide the data of risk evaluation for decision-makers and make the data increasingly exact, and prevent the security of grid in safe risk management, as well as improve the level and quality of grid of risk evaluation in rural areas.

\section{References}

1. Chen, W., Liao, J.: Decision support system and development. Tsinghua University Press, Beijing (2008)

2. Wu, Q.: Decision support system of flood control \& drought based on web. Computer Science 31(9), 271-277 (2004)

3. Li, Q., Chi, Z.: Regional power dispatching decision support system based on data warehouse. Automation of Electric Power Systems 28(12), 86-90 (2004)

4. Chen, L., Zhao, C.: Design \& implementation of intelligent decision support system for precision agriculture. Transaction of CSAE 18(2), 145-148 (2002)

5. Meng, J., Yang, G.: Research of macro decision support system for agriculture production. Agriculture System Science \& Comprehensive Studies 18(4), 293-297 (2002) 